\title{
Reciprocity Theorems for One-Way Wave Fields in Curvilinear Coordinate Systems*
}

\author{
Martijn Frijlink ${ }^{\dagger}$ and Kees Wapenaar ${ }^{\ddagger}$
}

\begin{abstract}
One-way wave equations conveniently describe wave propagation in media with discontinuous and/or rapid variations in one direction, but with smooth and slow variations in the complementary transverse directions. In the past, reciprocity theorems have been developed in terms of one-way wave fields. The boundaries of the integration volumes and the variations of the medium parameters must adhere to strict conditions. The variations must have the smoothness required by pseudodifferential operators, while the boundaries have to be flat. To extend the applicability to nonflat boundaries, this paper formulates one-way wave equations and corresponding reciprocity theorems in terms of curvilinear coordinates of the semiorthogonal (SO) type. In SO coordinate systems, one of the covariant basis vectors is orthogonal to the others, which can be nonorthogonal among each other. The same applies to the contravariant basis vectors. Furthermore, the orthogonal directions coincide; that is, the orthogonal co- and contravariant basis vectors coincide. SO coordinates are characterized by a local property of the basis vectors. An extra specification is necessary to make them conform in any way to nonflat boundaries. This can be done in terms of so-called lateral Cartesian (LC) coordinates. Cartesian coordinates are mapped to LC coordinates by applying an invertible transformation to one coordinate while keeping the others the same. LC coordinates are a straightforward means to describe or conform to nonflat boundaries. Applications of the extended reciprocity theorems include removal of multiple reflections, removal of complex propagation effects, wave field extrapolation, and synthesis of unrecorded data.
\end{abstract}

Key words. reciprocity theorems, curvilinear coordinates, one-way wave fields

AMS subject classifications. 35R $35,86 \mathrm{~A} 22$

DOI. $10.1137 / 080739185$

1. Introduction. The subsurface of the Earth has a predominantly layered structure; the variations with depth are much more rapid and contain more discontinuities than the variations in the horizontal directions. In reflection seismology the depth direction is the preferred or marching direction for wave propagation. To exploit this property, algorithms for processing seismic reflection measurements are often formulated in terms of up- and down-going wave fields. For media consisting of plane-parallel, homogeneous layers, such formulations are well established [12, 23].

Similar principles have been used in imaging and in more general inverse scattering, applied to electromagnetic and acoustic wave propagation $[9,22,28,34]$. These formalisms are not

\footnotetext{
* Received by the editors October 27, 2008; accepted for publication (in revised form) June 2, 2010; published electronically August 19, 2010. This research was funded by the Netherlands Research Centre for Integrated Solid Earth Science (ISES).

http://www.siam.org/journals/siims/3-3/73918.html

${ }^{\dagger}$ Department of Geotechnology, Delft University of Technology, 2600 GA Delft, The Netherlands. Current address: PGS, 2332 KG Leiden, The Netherlands (martijn.frijlink@pgs.com).

${ }^{\ddagger}$ Department of Geotechnology, Delft University of Technology, 2600 GA Delft, The Netherlands (C.P.A.Wapenaar @tudelft.nl).
} 


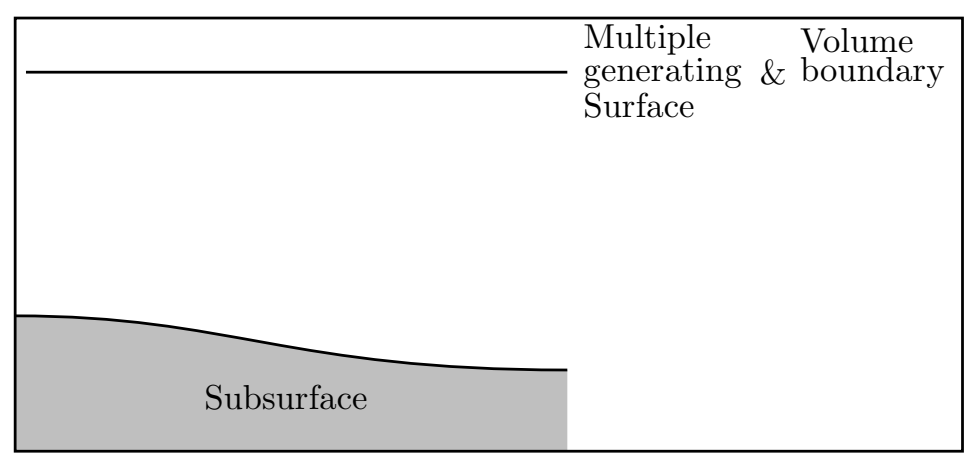

Figure 1. Volume boundary for removal of surface-related multiple reflections, based on the assumption that the pressure of the multiple contaminated state vanishes at the surface.

restricted to the horizontally layered media mentioned above, but the step of separation into up- and down-going wave fields must still be performed at flat boundaries. In this paper we first formulate wave field separation for nonflat boundaries, and second we formulate reciprocity theorems in terms of separated wave fields. The latter are well suited to serve as starting points for the formulation of the formalisms mentioned above for nonflat boundaries.

For laterally smooth media with discontinuities appearing only in the marching direction, De Hoop [11] and Wapenaar [27] factorized the full wave equation into coupled one-way wave equations for wave fields propagating in the marching direction and its opposite. As a second step Wapenaar formulated reciprocity theorems in terms of the one-way wave fields under flux normalization. In general, operators do not commute, which complicates solving the roots of the characteristic polynomial associated with a matrix operator. However, De Hoop and Wapenaar exploited the fact that the matrix operator governing the wave equation has a zero diagonal.

Reciprocity theorems for the total wave fields can handle the scattering response from arbitrarily varying media. The theorems are made up of volume integrals of products of pressure and particle velocity wave fields. The products can be interpreted as acoustic Poynting vectors of energy flow. The classic formulation of reciprocity theorems [5, 21] is based on two different types of wave fields, the scalar pressure and vector velocity. Wapenaar [27] adapted the classic formulation, in terms of two different types of wave fields, into a formulation in terms of one "intermediate" type: flux normalized one-way wave fields.

In their classic formulation, reciprocity theorems are particularly useful in exploiting Dirichlet or Neumann boundary conditions, that is, when the wave field or its derivative is known. Reciprocity theorems for one-way wave fields are designed to exploit boundary conditions in terms of incoming or outgoing wave fields. Similar principles can be derived from the two types of theorems, but with different physical assumptions and limitations.

A typical geophysical application of reciprocity theorems is the formulation of the processing steps of removal of surface-related multiple reflections $[1,5]$. The particular reciprocity theorem relates the multiple-free state and the multiple-contaminated state, and is derived from the idealized Dirichlet boundary condition stating that the pressure vanishes at the surface generating the multiples. The space outside the integration volume is assumed to be a vacuum, and the surface is assumed to be flat; see Figure 1. 


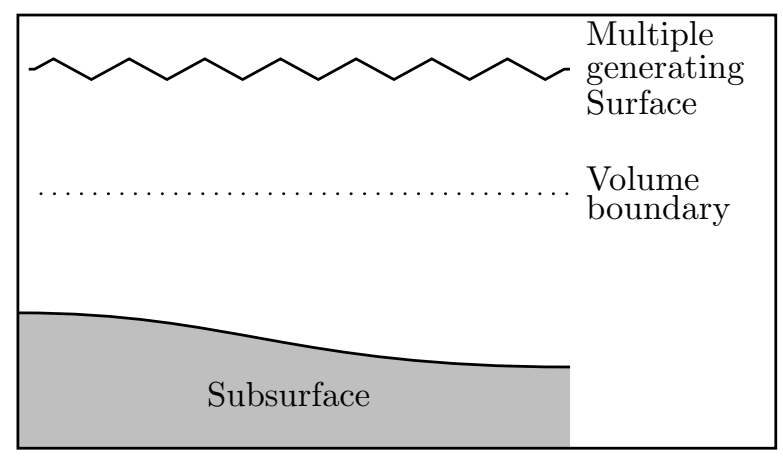

Figure 2. Volume boundary for removal of surface-related multiple reflections, based on the fact that the multiple-free state is purely up-going at and below the surface.

However, the multiple-free state obeys a more natural and less restrictive boundary condition: by definition the multiple-free state has no surface, so the wave field is purely outgoing; there is no incoming field, not just at the surface but also below it. As a result there is a degree of freedom in where to evaluate the boundary condition and the shape of the surface on which to evaluate it. Wapenaar [31] and Frijlink [6] evaluate the boundary condition at a horizontal level below the surface as in Figure 2; this horizontal level follows the global, flat trend of the surface but does not require specific knowledge about its shape or the medium above it.

Other geophysical applications include removal of the propagation effects of a complex part of the medium [7], wave field extrapolation [28], and synthesis of unrecorded data [31, 32]. Formulations of all applications can be derived from reciprocity theorems for the usual total wave fields; see [13] and [19]. A fundamental property that is exploited in each of the applications is source receiver reciprocity [5, 21]. A consequence of flux normalization is that transmission operators for up- and down-going wave fields obey source receiver reciprocity [30], while transmission operators for other normalizations lack this property.

The factorization of the wave equation deployed by De Hoop [11] and Wapenaar [27], or, rather, the diagonalization of the matrix operator governing the wave equation, requires lateral smoothness as the factorization involves the square root of the Helmholtz operator, which is a pseudodifferential operator; there are no smoothness restrictions on variations in the marching direction. If the layer structure deviates from being plane-parallel, then these operators will be applied erroneously to discontinuities related to the layer boundaries. The aim of this paper is to factorize the wave equation in coordinate systems that conform to nonplanar layer boundaries. The same smoothness condition for whose benefit we are trying to devise coordinate systems conforming to nonplanar layer boundaries also limits the amount of conformity that is possible. In general the layer boundaries themselves are not smooth enough for the application of pseudodifferential operators (see Van den Berg and De Hoop [25]). Exact conformation to layer boundaries is therefore impossible.

Instead, the hypersurfaces enclosing integration volumes, and the conformal coordinates describing them, are restricted to mimicking the smooth, large-scale trends of layer boundaries similar to the example of multiple removal described above. Similar to the plane-parallel case, said hypersurfaces have to be located in a part of the medium where the variations of the 
medium are smooth. The conforming hypersurface can never cross the actual layer boundary, so the integration volume either fully includes or entirely excludes the layer boundary.

Straightforward-to-specify conformal coordinate systems are those proposed by Haines and De Hoop [10]. We refer to these as lateral Cartesian (LC) coordinate systems, because they have the lateral coordinates in common with the standard Cartesian case. The third coordinate is, however, constant not at flat planes perpendicular to the lateral coordinates but instead at the nonflat hypersurfaces as described above following the global trend of major discontinuities in the medium parameter.

The main complication in diagonalizing the matrix operator mentioned earlier arises from the fact that its entries do not commute. As long as the diagonal entries are zero, a diagonalization is feasible. For nonorthogonal coordinates these diagonal entries become nonzero. The underlying reason is that nonorthogonal coordinates have metric tensors that are not diagonal. Hence the differential operators for such coordinates are more complex; see section 3.1 for a brief overview and, for example, Fung and Tong [8] for a comprehensive treatment.

The off-diagonal elements of the LC metric tensor destroy the zero diagonal of the matrix operator governing the acoustic wave equation that allowed the factorization proposed by De Hoop [11] and Wapenaar [27]; see section 4. In terms of so-called semiorthogonal (SO) coordinates the zero diagonal is conserved; see section 3.3 and also Sava and Fomel [18]. In sections 5 and 6 we exploit this conservation to show that for SO coordinates the diagonalization and subsequent manipulations of the wave equation as in [27] remain possible, finally leading to reciprocity theorems for flux normalized one-way wave fields. The defining property of SO coordinates does not offer a straightforward way to specify the coordinates themselves. We therefore tie SO to LC coordinates as described in section 3.3. If decomposition into incoming and outgoing components is required only at the volume boundaries but not in the interior, then the smoothness conditions of pseudodifferential operators have to be met only on those boundaries. Reciprocity theorems for this case will also be constructed in section 6 .

Tables 1(a) and 1(b) list symbols used in this paper, including a short description plus references to the defining expressions. Table 1(a) lists quantities related to coordinate systems (besides coordinates, also basis vectors and metric tensors), while Table 1(b) lists miscellaneous quantities.

2. Notation. In acoustic wave propagation the wave field quantities are the acoustic pressure $p$ and the particle velocity $\mathbf{v}=v^{l} \mathbf{e}_{l}$. Here $\left\{\mathbf{e}_{1}, \mathbf{e}_{2}, \mathbf{e}_{3}\right\}$ are the Cartesian basis vectors. In this paper we use the summation convention to sum over repeated indices occurring twice in a product, where one instance is a subscript and the other a superscript. Furthermore, we use roman indices for summation over $\{1,2,3\}$ and Greek indices for summation over just $\{1,2\}$. Note that the directional indices of the vector components $v^{l}$ appear as superscripts; this is an example of the convention, employed in this paper, that vector components with superscripts correspond to basis vectors with subscripts. Both $p$ and $\mathbf{v}$ are functions of time $t$ and position $\mathbf{x}=\left(x_{1}, x_{2}, x_{3}\right)^{t}$; the ${ }^{t}$-superscript indicates a transposed vector.

We often isolate dependency on the coordinate $x_{3}$ from the other two coordinates as in $p(\mathbf{x})=p\left(\boldsymbol{x}_{L}, x_{3}\right)$, with $\boldsymbol{x}_{L}=\left(x_{1}, x_{2}\right)^{t}$. Curvilinear coordinates will be separated in a similar fashion.

The function $P(\mathbf{x}, \omega)$ denotes the complex-valued, space-frequency counterpart of the real- 
Table 1(a)

Table of coordinate-related symbols.

\begin{tabular}{|c|c|c|}
\hline Symbol & Description & $\begin{array}{l}\text { Defining } \\
\text { equations }\end{array}$ \\
\hline$\xi$ & $=\left(\xi_{1}, \xi_{2}, \xi_{3}\right)$ curvilinear coordinates & $(3.1)$ \\
\hline $\boldsymbol{\xi}_{L}$ & $=\left(\xi_{1}, \xi_{2}\right)$ lateral curvilinear coordinates & \\
\hline$\tilde{\xi}$ & lateral Cartesian (LC) coordinates & $(3.10)$ \\
\hline$\underline{\xi}$ & semiorthogonal ( $\mathrm{SO}$ ) coordinates & $(3.15)$ \\
\hline$f$ & function mapping $\tilde{\xi}_{3}$ to $x_{3}$ & $(3.10)$ \\
\hline $\mathrm{g}, \tilde{\boldsymbol{g}}, \underline{g}$ & $\begin{array}{l}\text { Metric tensor, elements } g_{i j}=\mathbf{g}_{i} \cdot \mathbf{g}_{j} . \\
\text { The SO metric tensor is blockdiagonal: } \\
\underline{\boldsymbol{g}}=\left(\begin{array}{ccc}\underline{g}_{11} & \underline{g}_{12} & 0 \\
\underline{g}_{21} & \underline{g}_{22} & 0 \\
0 & \underline{g}_{33}\end{array}\right) . \\
\text { The LC metric tensor } \tilde{\boldsymbol{g}} \text { is a full } \\
3 \times 3 \text { matrix. }\end{array}$ & $\begin{array}{l}(3.6) \\
(3.15)\end{array}$ \\
\hline $\mathbf{g}_{i}, \tilde{\boldsymbol{g}}_{i}, \underline{\boldsymbol{g}}_{i}$ & $\begin{array}{l}\text { Covariant basis vectors. LC instances } \\
\text { make up the Jacobian matrix } \\
\left(\tilde{\boldsymbol{g}}_{1}, \tilde{\boldsymbol{g}}_{2}, \tilde{\boldsymbol{g}}_{3}\right)=\left(\begin{array}{ccc}1 & 0 & 0 \\
0 & 1 & 0 \\
\tilde{\xi}_{\tilde{\xi}_{1}} f & \partial_{\tilde{\xi}_{2}} f & \partial_{\tilde{\xi}_{3}} f\end{array}\right) . \\
\text { The orthogonality relations implied by } \\
\text { the SO metric tensor } \underline{\boldsymbol{g}} \text { characterize } \\
\text { SO basis vectors. }\end{array}$ & $\begin{array}{l}(3.2) \\
(3.12)\end{array}$ \\
\hline $\mathbf{g}^{i}, \tilde{\boldsymbol{g}}^{i}, \underline{\boldsymbol{g}}^{i}$ & contravariant basis vectors & $(3.3)$ \\
\hline$g, \tilde{g}, \underline{g}$ & $=\operatorname{det} \mathbf{g}$ & \\
\hline $\mathbf{e}_{k}$ & Cartesian basis vectors & \\
\hline $\mathbf{n}, \tilde{\boldsymbol{n}}, \underline{\boldsymbol{n}}$ & $\begin{array}{l}\text { normal vectors to surfaces of constant } \\
\xi_{3}, \tilde{\xi}_{3}, \text { or } \underline{\xi}_{3}\end{array}$ & $(3.5)$ \\
\hline
\end{tabular}

valued, space-time function $p(\mathbf{x}, t)$. The two are related to each other by the Fourier transform

$$
P(\mathbf{x}, \omega)=\int_{-\infty}^{+\infty} p(\mathbf{x}, t) \exp (-j \omega t) d t
$$

and its inverse

$$
p(\mathbf{x}, t)=\pi^{-1} \Re\left[\int_{0}^{+\infty} P(\mathbf{x}, \omega) \exp (j \omega t) d \omega\right],
$$

where $j$ is the imaginary unit and the symbol $\Re$ means taking the real part. Throughout this paper we consider positive frequencies only. For notational convenience we suppress the 
Table 1(b)

Table of symbols.

\begin{tabular}{|c|c|c|}
\hline Symbol & $\overline{\text { Description }}$ & $\begin{array}{l}\text { Defining } \\
\text { equations }\end{array}$ \\
\hline$\omega$ & angular frequency & $(2.1)$ \\
\hline$\underline{\hat{\Lambda}}$ & one-way propagation operator & $(5.2),(5.7)$ \\
\hline$\underline{\hat{\Theta}}$ & one-way scattering operator & $(5.5)$ \\
\hline$\rho$ & mass density & \\
\hline$\hat{\mathbf{A}}$ & wave operator & $\begin{array}{l}(4.9) \\
(4.14 \mathrm{a})-(4.14 \mathrm{c}) \\
\end{array}$ \\
\hline$\underline{\hat{A}}$ & Helmholtz-type operator & $(5.6)$ \\
\hline$\underline{\hat{\mathbf{B}}}$ & one-way wave operator & $(5.4),(5.5)$ \\
\hline$\hat{D}_{\nu}$ & scaled spatial derivative & $(4.10)$ \\
\hline D & $\begin{array}{l}2 \text {-vector with force and volume injection } \\
\text { sources }\end{array}$ & $(4.8)$ \\
\hline $\mathbf{J}$ & $=\left(\begin{array}{cc}1 & 0 \\
0 & -1\end{array}\right)$ & $(5.7)$ \\
\hline $\mathbf{K}$ & $=\left(\begin{array}{ll}0 & 1 \\
1 & 0\end{array}\right)$ & $(4.16)$ \\
\hline$K$ & compression modulus & \\
\hline$\underline{\hat{\mathbf{L}}}^{ \pm 1}$ & $\begin{array}{l}\text { (de)composition operator, } \\
\text { only for SO coordinates }\end{array}$ & $(5.2),(5.8),(5.9)$ \\
\hline$\underline{\hat{L}}$ & basic element of $\underline{\hat{\mathbf{L}}}^{ \pm 1}$ & $(5.10)$ \\
\hline $\mathbf{N}$ & $=\left(\begin{array}{cc}0 & 1 \\
-1 & 0\end{array}\right)$ & $(4.15)$ \\
\hline$P, p$ & pressure & $(2.1),(2.2)$ \\
\hline$\underline{P}$ & $\begin{array}{l}\text { 2-vector with flux normalized, } \\
\text { up- and down-going wave fields }\end{array}$ & $(5.4)$ \\
\hline$\Re$ & real part of complex number & \\
\hline$\underline{S}$ & $\begin{array}{l}\text { 2-vector with flux normalized, } \\
\text { up- and down-going source fields }\end{array}$ & $(5.4)$ \\
\hline $\mathbf{V}, V^{k}, v^{l}$ & (components of) the particle velocity & \\
\hline$V^{\mathbf{n}}$ & inner product $\mathbf{n} \cdot \mathbf{V}$ & $(4.3),(4.4)$ \\
\hline $\mathbf{Z}$ & 2 -vector of $P$ and $V^{\mathbf{n}}$ & $(4.8)$ \\
\hline
\end{tabular}

Copyright (C) by SIAM. Unauthorized reproduction of this article is prohibited. 


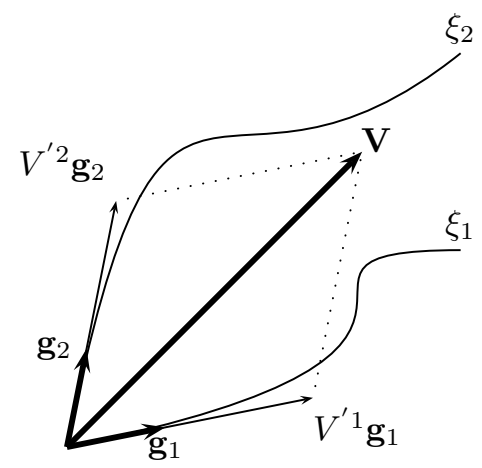

Figure 3. Covariant basis vectors $\mathbf{g}_{1}$ and $\mathbf{g}_{2}$. The vector $\mathbf{g}_{k}$ is tangential to the curve resulting from varying only $\xi_{k}$.

angular frequency $\omega$ from here on. With this convention the pressure appears as $P(\mathbf{x})$ and the components of the particle velocity as $V^{k}(\mathbf{x})$.

From section 4 on we will use adjoint vectors in addition to transposed vectors. For a given operator $\hat{R}$, the transposed $\hat{R}^{t}$ and adjoint $\hat{R}^{\dagger}$ are defined, respectively, by

$$
\begin{aligned}
\int_{\mathbb{R}^{2}} w\left(\boldsymbol{x}_{L}\right) \hat{R}^{t} u\left(\boldsymbol{x}_{L}\right) d^{2} \boldsymbol{x}_{L} & =\int_{\mathbb{R}^{2}} \hat{R} w\left(\boldsymbol{x}_{L}\right) u\left(\boldsymbol{x}_{L}\right) d^{2} \boldsymbol{x}_{L}, \\
\int_{\mathbb{R}^{2}} w^{*}\left(\boldsymbol{x}_{L}\right) \hat{R}^{\dagger} u\left(\boldsymbol{x}_{L}\right) d^{2} \boldsymbol{x}_{L} & =\int_{\mathbb{R}^{2}} \hat{R} w^{*}\left(\boldsymbol{x}_{L}\right) u\left(\boldsymbol{x}_{L}\right) d^{2} \boldsymbol{x}_{L} .
\end{aligned}
$$

For this paper three special cases are important: symmetric operators obey $\hat{R}^{t}=\hat{R}$, skew symmetric operators obey $\hat{R}^{t}=-\hat{R}$, and self-adjoint operators obey $\hat{R}^{\dagger}=\hat{R}$. For example, Berezansky, Sheftel, and Us $[2,3]$ give more details on linear integral operators.

\section{Curvilinear coordinates.}

3.1. Some general properties. We assume that there is a one-to-one, reversible mapping between Cartesian and curvilinear coordinates $\boldsymbol{\xi}=\left(\xi_{1}, \xi_{2}, \xi_{3}\right)$, according to

$$
\xi_{k}=\xi_{k}\left(x_{1}, x_{2}, x_{3}\right) \quad \text { and } \quad x_{l}=x_{l}\left(\xi_{1}, \xi_{2}, \xi_{3}\right)
$$

for $k, l=\{1,2,3\}$. Whereas Cartesian coordinates have one set of basis vectors, curvilinear coordinates in general have two; covariant and contravariant basis vectors are defined, respectively, by

$$
\begin{aligned}
\mathbf{g}_{k} & =\left(\partial x_{i} / \partial \xi_{k}\right) \mathbf{e}_{i} \quad \text { and } \\
\mathbf{g}^{l} & =\left(\partial \xi_{l} / \partial x_{i}\right) \mathbf{e}_{i},
\end{aligned}
$$

where $i \in\{1,2,3\}$; summation over $i$ is implied in both (3.2) and (3.3). The covariant basis vector $\mathbf{g}_{1}$ is tangent to curves characterized by constant $\xi_{2,3}$, whereas the contravariant basis vector $\mathbf{g}^{1}$ is perpendicular to a hypersurface characterized by constant $\xi_{1}$. A two-dimensional example is shown in Figures 3 and 4 . The contravariant basis vectors displayed in Figure 4 


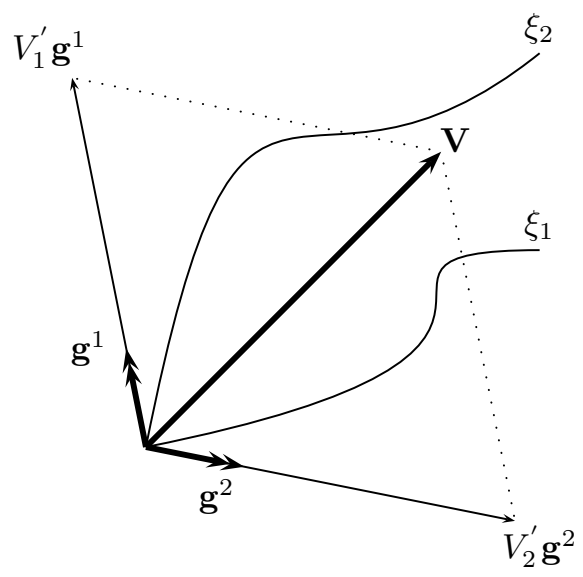

Figure 4. Contravariant basis vectors $\mathbf{g}^{1}$ and $\mathbf{g}^{2}$ with double arrowheads. The vector $\mathbf{g}^{k}$ is orthogonal to the surface of constant $\xi_{k}$.

have double arrowheads. The covariant basis vectors are in general not orthogonal with respect to each other, and neither are the contravariant basis vectors. However, by definition each individual contravariant basis vector $\mathbf{g}^{l}$ is orthogonal to the covariant basis vectors $\mathbf{g}_{k}$ if $l \neq k$, and if $l$ does equal $k$, then their inner product is unity. Hence,

$$
\mathbf{g}^{l} \cdot \mathbf{g}_{k}=\delta_{l k} .
$$

The first of two pivotal quantities in this paper is the cross-product defined by

$$
\mathbf{n}=\mathbf{g}_{1} \times \mathbf{g}_{2} .
$$

From (3.4) it can be seen that the vector $\mathbf{n}$ is parallel to $\mathbf{g}^{3}$, i.e., is perpendicular to a surface of constant $\xi_{3}$. However, $\mathbf{n}$ will turn out to be more convenient to use in reciprocity theorems. The second pivotal quantity is the metric tensor $\mathbf{g}$, or rather its inverse. These are $3 \times 3$ symmetric matrices. The nine matrix-elements of the metric tensor are defined by

$$
g_{k l}=\mathbf{g}_{k} \cdot \mathbf{g}_{l} .
$$

Expressing the gradient and divergence in partial derivatives $\partial / \partial \xi_{l}$ requires the determinant $g=\operatorname{det} \mathbf{g}>0$ and the inverse $\mathbf{g}^{-1}$ of the metric tensor whose elements are given, respectively, by

$$
g^{k l}=\mathbf{g}^{k} \cdot \mathbf{g}^{l} .
$$

Equation (3.4) can be used to establish that the matrix made up by the elements $g^{k l}$ and that made up by $g_{k l}$ are indeed each other's inverse. See also Fung and Tong [8].

Scalar quantities in terms of curvilinear coordinates have a prime $\left({ }^{\prime}\right)$ superscript attached; their Cartesian counterparts do not. In the case of pressure $P$ the two different functions are related by

$$
P\left(x_{1}, x_{2}, x_{3}\right)=P^{\prime}\left(\xi_{1}, \xi_{2}, \xi_{3}\right)
$$




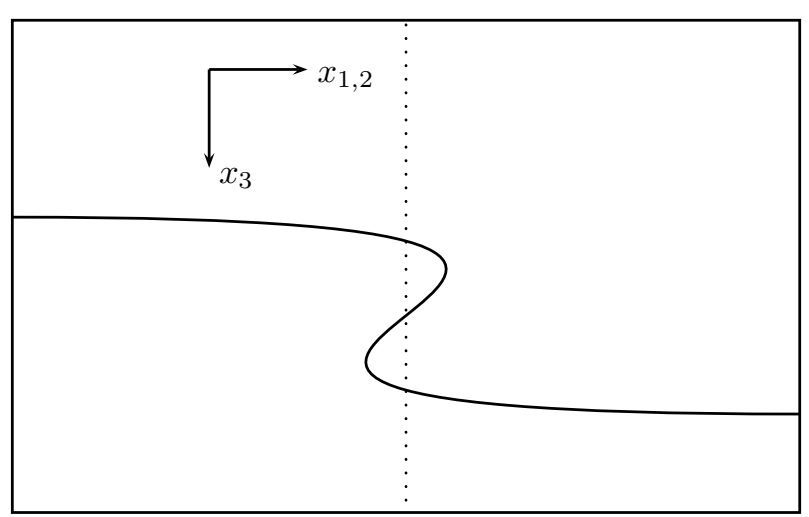

Figure 5. Example of a multivalued and therefore irreversible mapping between Cartesian and LC coordinates. The vertical dashed line is characterized by constant values of $x_{1}$ and $x_{2}$. If we attach a constant value of $\xi_{3}$ to the hypersurface, then three different values of $x_{3}$ are mapped to the single $\xi_{3}$-value.

For vector quantities such as the particle velocity $\mathbf{V}$, a relation similar to (3.8) holds only for the entire vector but not for the individual scalar components; i.e.,

$$
\mathbf{V}=V^{i} \mathbf{e}_{i}=V^{\prime k} \mathbf{g}_{k},
$$

whereas $V^{\prime i}\left(\xi_{1}, \xi_{2}, \xi_{3}\right) \neq V^{i}\left(x_{1}, x_{2}, x_{3}\right)$ in general.

In this paper we will use two particular types of curvilinear coordinates, lateral Cartesian (LC) and semiorthogonal (SO), introduced, respectively, in sections 3.2 and 3.3.

3.2. Lateral Cartesian coordinates. The first type of curvilinear coordinate that we consider is that of LC coordinates. The lateral coordinates are identical to their Cartesian counterparts, but the third LC coordinate is assumed to be constant at hypersurfaces conforming to the smooth, large-scale trends of layer boundaries. LC coordinates and functions or operators depending on them will be identified by an overlying tilde, e.g., $\tilde{\xi}$ :

$$
x_{1}=\tilde{\xi}_{1}, \quad x_{2}=\tilde{\xi}_{2}, \quad x_{3}=f\left(\tilde{\xi}_{1}, \tilde{\xi}_{2}, \tilde{\xi}_{3}\right) .
$$

Like Haines and De Hoop [10] we let $f$ be a function chosen such that nonflat hypersurfaces are mapped to surfaces of constant $\tilde{\xi}_{3}$. The coordinate mapping implied by (3.10) is reversible, as long as the angle between each tangent of the hypersurfaces and the $\left(x_{1}, x_{2}\right)$-plane is less than $90^{\circ}$. However, hypersurfaces such as those shown in Figure 5 with tangents at $90^{\circ}$ or more map more than one value of $x_{3}$ to one value of $\tilde{\xi}_{3}$. Such hypersurfaces therefore cannot be described by (3.10). A further limitation on the class of hypersurface that this approach can handle is due to the involvement of square and fourth order roots of the Helmholtz operator, both pseudodifferential operators which require smooth variations as a function of $x_{1}$ and $x_{2}$; see sections 5 and 6 . As indicated in the introduction, one can therefore put the volume boundaries described by these hypersurfaces only where the variations of the medium obey this requirement of smoothness.

The nontrivial part of inverting the coordinate transformation described by (3.10) requires inverting $f$ for constant $x_{1}$ and $x_{2}$. We describe this inversion for mapping $x_{3}$ to $\xi_{3}$ by 


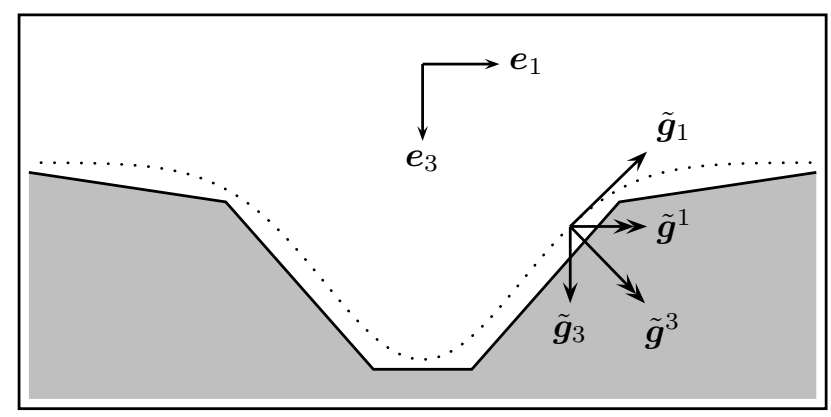

Figure 6. Example of $L C$ covariant basis vectors (single arrowhead) and contravariant basis vectors (double arrowhead) for a Gaussian-shaped volume boundary, described by $f=\tilde{\xi}_{3}+\exp \left(-\tilde{\xi}_{1}^{2} / 2\right)$. The "hypersurface" of constant $\tilde{\xi}_{3}$ is represented by the dotted Gaussian curve.

$f_{\text {inv }}$. With this notation the inverse of the coordinate transformation from Cartesian to LC coordinates is

$$
\tilde{\xi}_{1}=x_{1}, \quad \tilde{\xi}_{2}=x_{2}, \quad \tilde{\xi}_{3}=f_{\text {inv }}\left(x_{1}, x_{2}, x_{3}\right) .
$$

In the shape of column vectors, the covariant basis vectors for LC coordinates make up the Jacobian matrix

$$
\left(\tilde{\boldsymbol{g}}_{1}, \tilde{\boldsymbol{g}}_{2}, \tilde{\boldsymbol{g}}_{3}\right)=\left(\begin{array}{ccc}
1 & 0 & 0 \\
0 & 1 & 0 \\
\partial_{\tilde{\xi}_{1}} f & \partial_{\tilde{\xi}_{2}} f & \partial_{\tilde{\xi}_{3}} f
\end{array}\right)
$$

The corresponding contravariant basis vectors are

$$
\left(\tilde{\boldsymbol{g}}^{1}, \tilde{\boldsymbol{g}}^{2}, \tilde{\boldsymbol{g}}^{3}\right)=\left(\begin{array}{ccc}
1 & 0 & \partial_{x_{1}} f_{i n v} \\
0 & 1 & \partial_{x_{2}} f_{i n v} \\
0 & 0 & \partial_{x_{3}} f_{i n v}
\end{array}\right) .
$$

Alternatively the contravariant basis vectors can be derived from (3.4) and (3.12). For a Gaussian-shaped volume boundary described by $f=\tilde{\xi}_{3}+\exp \left(-\tilde{\xi}_{1}^{2} / 2\right)$, Figure 6 shows the covariant and contravariant basis vectors in a plane of constant $x_{2}$. The contravariant basis vectors have double arrowheads, the covariant basis vectors have single arrowheads, and the "hypersurface" of constant $\tilde{\xi}_{3}$ is represented by the dotted Gaussian curve.

In terms of $\mathrm{LC}$ coordinates, the normal direction $\mathbf{n}$ reads as

$$
\tilde{\boldsymbol{n}}=\left(-\partial_{\tilde{\xi}_{1}} f,-\partial_{\tilde{\xi}_{2}} f, 1\right)^{t}
$$

where the superscript $t$ denotes transposition. The vector $\tilde{\boldsymbol{n}}$ is the direction perpendicular to surfaces of constant $\xi_{3}$ [10]. It is taken as the direction of decomposition, similar to $\mathbf{e}_{3}$ in Cartesian coordinates. But unlike its Cartesian analogue, $\tilde{\boldsymbol{n}}$ has a position-dependent direction and length greater than one. In section 3.3 we will choose SO basis vectors such that $\mathbf{n}$ in SO coordinates will coincide with $\tilde{\boldsymbol{n}}$ in LC coordinates. For this reason we also 
attach the meaning of marching direction to $\mathbf{n}$ defined by (3.5), although it is based on the more arbitrary coordinates of (3.1) rather than on SO or LC coordinates. The SO coordinates tied to LC coordinates will be used in section 6 .

Note that, owing to the lower triangular structure of the Jacobian matrix given by (3.12), the determinant $\tilde{g}$ of the metric tensor for LC coordinates equals

$$
\tilde{g}=\left(\partial_{\tilde{\xi}_{3}} f\right)^{2},
$$

and that both the metric tensor $\tilde{\boldsymbol{g}}$ and its inverse $\tilde{\boldsymbol{g}}^{-1}$ are full $3 \times 3$ matrices. This latter property sets them apart from SO coordinates.

3.3. Semiorthogonal coordinates. The second particular type of curvilinear coordinate used in this paper is that of SO coordinates; these coordinates and functions or operators depending on them are identified by a bar underneath, e.g., $\xi$. Their main advantage is a reduction of analytical complexity, which will be exploited in section 5 for directional decomposition into one-way, flux normalized wave fields. SO coordinates do not have compact expressions like (3.10) in terms of Cartesian coordinates. Rather, the reduced complexity for SO coordinates is due to the zeros appearing in the metric tensor.

In geophysical literature Sava and Fomel [18] used the term SO for coordinates characterized by a block diagonal metric tensor

$$
\underline{\boldsymbol{g}}=\left(\begin{array}{ccc}
\underline{g}_{11} & \underline{g}_{12} & 0 \\
\underline{g}_{21} & \underline{g}_{22} & 0 \\
0 & 0 & \underline{g}_{33}
\end{array}\right) .
$$

Remember that (3.4) implies that the contravariant basis vector $\boldsymbol{g}^{3}$ is by construction orthogonal to the covariant basis vectors $\underline{\boldsymbol{g}}_{1,2}$. The zeros in (3.15) express that the lateral basis vectors $\underline{\boldsymbol{g}}_{1,2}$ are also orthogonal to the basis vectors $\underline{\boldsymbol{g}}_{3}$, and consequentially $\underline{\boldsymbol{g}}^{3}$ and $\underline{\boldsymbol{g}}_{3}$ are parallel. The orthogonality relations implied in (3.15) set SO basis vectors apart from LC basis vectors. The latter are in general nonorthogonal; that is, $\tilde{g}_{13} \neq 0$ and $\tilde{g}_{23} \neq 0$. The SO inverse metric tensor $\underline{\boldsymbol{g}}^{-1}$ has the same block diagonal structure as $\underline{\boldsymbol{g}}$,

$$
\underline{\boldsymbol{g}}^{-1}=\left(\begin{array}{ccc}
\underline{g}^{11} & \underline{g}^{12} & 0 \\
\underline{g}^{21} & \underline{g}^{22} & 0 \\
0 & 0 & \underline{g}^{33}
\end{array}\right),
$$

so the contravariant basis vectors $\underline{\boldsymbol{g}}^{1,2}$ are similarly orthogonal to $\underline{\boldsymbol{g}}^{3}$ and $\underline{\boldsymbol{g}}_{3}$. In section 4 it will become clear that SO coordinates allow a simpler formulation of the wave equation than LC coordinates. However, SO coordinates are defined by the form of the associated metric tensor, and the actual coordinates are still to be defined. In section 6 we will use a special choice of SO coordinates in terms of LC coordinates. For constant $\tilde{\xi}_{3}$ we take covariant basis vectors

$$
\underline{\boldsymbol{g}}_{1}=\tilde{\boldsymbol{g}}_{1}, \quad \underline{\boldsymbol{g}}_{2}=\tilde{\boldsymbol{g}}_{2}, \quad \text { and } \quad \underline{\boldsymbol{g}}_{3}=\underline{\boldsymbol{n}}=\tilde{\boldsymbol{n}} .
$$

Since $\tilde{\boldsymbol{n}}$ is by construction orthogonal to $\tilde{\boldsymbol{g}}_{1,2}$ (recall the definition (3.5)), the basis vectors in (3.17) satisfy the defining property of SO coordinates, (3.15). For the Gaussian-shaped 


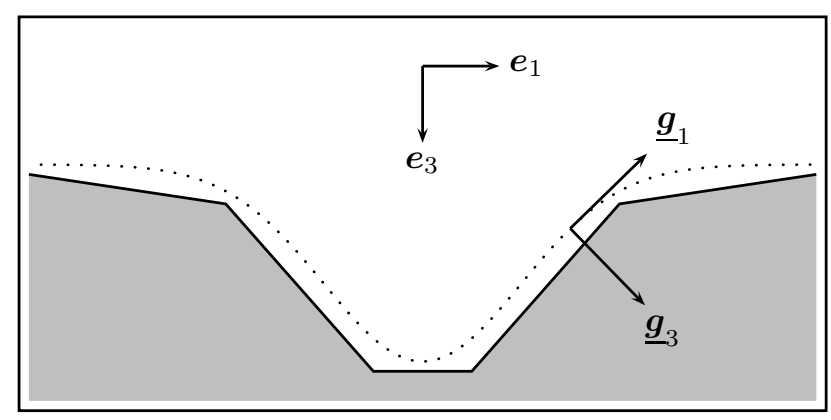

Figure 7. Example of $S O$ covariant basis vectors for a Gaussian-shaped volume boundary.

volume boundary shown in Figure 6 , the resulting covariant SO basis vectors are displayed in Figure 7. The metric tensor and determinant corresponding to the basis vectors of (3.17) become, respectively,

$$
\underline{\boldsymbol{g}}=\left(\begin{array}{ccc}
\left|\tilde{\boldsymbol{g}}_{1}\right|^{2} & \tilde{\boldsymbol{g}}_{2} \cdot \tilde{\boldsymbol{g}}_{1} & 0 \\
\tilde{\boldsymbol{g}}_{1} \cdot \tilde{\boldsymbol{g}}_{2} & \left|\tilde{\boldsymbol{g}}_{2}\right|^{2} & 0 \\
0 & 0 & |\tilde{\boldsymbol{n}}|^{2}
\end{array}\right)
$$

and $\underline{g}=|\tilde{\boldsymbol{n}}|^{4}$; recall (3.12) and (3.13).

4. The wave equation in curvilinear coordinates. In section 6 we will define reciprocity theorems for flux normalized wave fields based on the acoustic Poynting vector $P^{\prime} \mathbf{V}$ integrated over a hypersurface of constant $\xi_{3}$; recall (3.10). The basic form of these integrals is

$$
\int_{\xi_{3}=c} P^{\prime} \mathbf{V} \cdot\left(\mathbf{g}_{1} \times \mathbf{g}_{2}\right) d \xi_{1} d \xi_{2}=\int_{\xi_{3}=c} P^{\prime} V^{\mathbf{n}} d^{2} \boldsymbol{\xi}_{L}
$$

where $\boldsymbol{\xi}_{L}=\left(\xi_{1}, \xi_{2}\right)$ and

$$
V^{\mathbf{n}}=\mathbf{V} \cdot \mathbf{n}
$$

also recall the definition (3.5) of $\mathbf{n}$. In this section we will cast the wave equation in terms of $P$ and $V^{\mathbf{n}}$, which will serve as the basis for formulating directional decomposition in section 5 .

Because $\mathbf{n}$ is not a unit vector, the function $V^{\mathbf{n}}$ is merely proportional to the particle velocity in the direction normal to a surface of constant $\xi_{3}$ but not equal to it. Its usefulness arises from the compact form it induces in reciprocity theorems based on (4.1). However, the wave equation in curvilinear coordinates uses the three components $V^{\prime i}$. To express $V^{\mathbf{n}}$ in terms of these components we first substitute (3.2) into (3.9), then substitute the result into (4.2), and finally evaluate its inner product. Due to the definition of $\mathbf{n},(3.5)$, only the inner product $\mathbf{n} \cdot \mathbf{g}_{3}$ is nonzero, so that

$$
V^{\mathbf{n}}=\left(\mathbf{n} \cdot \mathbf{g}_{3}\right) V^{\prime 3}
$$

For LC coordinates (3.12)-(3.14) allow $V^{\mathbf{n}}$ to be expressed as $\tilde{V}^{\mathbf{n}}=\tilde{g}^{1 / 2} \tilde{V}^{\prime 3}$. The LC-based choice of SO coordinates implied by (3.17) similarly results in $\underline{\boldsymbol{V}}^{\mathbf{n}}=\underline{g}^{1 / 2} \underline{V}^{3}$. Instead of (4.2) or (4.3) we will therefore work with

$$
V^{\mathbf{n}}=g^{1 / 2} V^{\prime 3} .
$$

Copyright (c) by SIAM. Unauthorized reproduction of this article is prohibited. 
The total wave field is completely determined by the pressure $P^{\prime}$ and the scalar particle velocity $V^{\mathbf{n}}$. So without loss of generality the equations governing wave propagation can be expressed exclusively in terms of these two quantities without the need of the lateral velocity components $V^{\prime 1}$ and $V^{\prime 2}$. In the remainder of this paper quantities that depend on Cartesian coordinates are not necessary anymore, so we are going to omit prime superscripts from here on.

Given Fung's expressions of the gradient and divergence in curvilinear coordinates [8], the basic equations for wave propagation in acoustic, lossless media are

$$
\begin{aligned}
g^{k l} \partial_{\xi_{l}} P+j \omega \rho V^{k} & =F^{k}, \\
g^{-1 / 2} \partial_{\xi_{k}}\left(g^{1 / 2} V^{k}\right)+j \omega K^{-1} P & =Q
\end{aligned}
$$

for $k, l=\{1,2,3\}$; here the medium is described by the density $\rho$ and compression modulus $K$, while $F^{k}$ and $Q$ are, respectively, the force and volume injection sources. For our purpose, the crucial difference between (4.5) and (4.6) on the one hand and their Cartesian counterparts on the other is that in each component of (4.5) all three spatial derivatives occur. The process and final result of eliminating two components of the particle velocity $\mathbf{V}$ from the system of (4.5) and (4.6) is therefore more complex than in the Cartesian case.

Our goal is to express the system of (4.5) and (4.6) in the form

$$
\begin{aligned}
& \partial_{\xi_{3}} \mathbf{Z}+j \omega \hat{\mathbf{A}} \mathbf{Z}=\mathbf{D}, \\
& \text { where } \mathbf{Z}^{t}=\left(P, V^{\mathbf{n}}\right), \\
& \text { and } \quad \hat{\mathbf{A}}=\left(\begin{array}{ll}
\hat{A}_{11} & \hat{A}_{12} \\
\hat{A}_{21} & \hat{A}_{22}
\end{array}\right) .
\end{aligned}
$$

The 2-vector $\mathbf{D}$ contains all references to the source functions $Q$ and $F^{k}$. For the purpose of this paper it is not essential to have an explicit expression for $\mathbf{D}$. In the remainder of this section the source functions will therefore be set to zero. We refer to $\hat{\mathbf{A}}$ as the wave operator; its properties are the pivotal elements of this paper. The primary objective of formulating (4.7) is to gather all occurrences of lateral partial derivatives $\partial_{\xi_{\nu}}$ (for $\nu=1,2$ ) in one single matrix operator.

Before starting the actual elimination process, we need to make two remarks. For algebraic convenience a factor $-(j \omega)^{-1}$ is absorbed into the lateral partial derivatives:

$$
\hat{D}_{\nu}=-(j \omega)^{-1} \partial_{\xi_{\nu}} .
$$

The operators $\hat{D}_{\nu}$ have the interpretation of lateral slowness operators. These operators are skew symmetric and self-adjoint; i.e.,

$$
\hat{D}_{\nu}^{t}=-\hat{D}_{\nu} \quad \text { and } \quad \hat{D}_{\nu}^{\dagger}=\hat{D}_{\nu} .
$$

First we eliminate $V^{\prime 3}$ from (4.5) and (4.6) in favor of $V^{\mathbf{n}}$ with (4.4):

$$
\begin{aligned}
g^{33} \partial_{\xi_{3}} P-j \omega g^{3 \nu} \hat{D}_{\nu} P+\frac{j \omega \rho}{g^{1 / 2}} V^{\mathbf{n}} & =0, \\
g^{\mu 3} \partial_{\xi_{3}} P-j \omega g^{\mu \nu} \hat{D}_{\nu} P+j \omega \rho V^{\mu} & =0, \\
\partial_{\xi_{3}} V^{\mathbf{n}}+\frac{j \omega g^{1 / 2}}{K} P-j \omega \hat{D}_{\mu}\left(g^{1 / 2} V^{\mu}\right) & =0 .
\end{aligned}
$$

Copyright (c) by SIAM. Unauthorized reproduction of this article is prohibited. 
The elements $\hat{A}_{11}$ and $\hat{A}_{12}$ can be found by inspection from (4.12a). Elimination of $V^{\mu}$ from (4.12c) requires substitution of $(4.12 \mathrm{~b})$, but this still contains terms proportional to $\partial_{\xi_{3}} P$. We therefore first eliminate these terms from (4.12b) with the preceding substitution of (4.12a):

$$
g^{1 / 2} V^{\mu}=\frac{g^{1 / 2}}{\rho}\left(g^{\mu \nu}-\frac{g^{\mu 3} g^{3 \nu}}{g^{33}}\right) \hat{D}_{\nu} P+\frac{g^{\mu 3}}{g^{33}} V^{\mathbf{n}} .
$$

After substitution of (4.13) into (4.12c) we can also find the other elements of $\hat{\mathbf{A}}$ by inspection. Together with their symmetry properties the elements of $\hat{\mathbf{A}}$ read as

$$
\begin{aligned}
\hat{A}_{11} & =-\frac{g^{3 \nu}}{g^{33}} \hat{D}_{\nu}=-\hat{A}_{22}^{t}=\hat{A}_{22}^{\dagger}, \\
\hat{A}_{12} & =\frac{\rho}{g^{1 / 2} g^{33}}=\hat{A}_{12}^{t}=\hat{A}_{12}^{\dagger}, \\
\hat{A}_{21} & =\frac{g^{1 / 2}}{K}-\hat{D}_{\mu}\left[\frac{g^{1 / 2}}{\rho}\left(g^{\mu \nu}-\frac{g^{\mu 3} g^{3 \nu}}{g^{33}}\right) \hat{D}_{\nu} \cdot\right] \\
& =\hat{A}_{21}^{t}=\hat{A}_{21}^{\dagger} .
\end{aligned}
$$

Given the symmetry of the inverse metric tensor $g^{k l}$ and the fact that it is real-valued, the symmetry of $\hat{A}_{21}$ follows from the fact that $\hat{D}_{\nu}$ is skew symmetric and self-adjoint. The fact that $\hat{A}_{21}$ is self-adjoint is our motivation to work in lossless media. Collectively the symmetry relations implied by (4.14a)-(4.14c) can be expressed as the so-called symplectic property; that is, the matrix operator $\hat{\mathbf{A}}$ and its transposed $\hat{\mathbf{A}}^{t}$ are related by the expression

$$
\hat{\mathbf{A}}^{t} \mathbf{N}=-\mathbf{N} \hat{\mathbf{A}} \quad \text { with } \quad \mathbf{N}=\left(\begin{array}{cc}
0 & 1 \\
-1 & 0
\end{array}\right) .
$$

A similar relation holds for the adjoint matrix operator $\hat{\mathbf{A}}^{\dagger}$ :

$$
\hat{\mathbf{A}}^{\dagger} \mathbf{K}=\mathbf{K} \hat{\mathbf{A}} \quad \text { with } \quad \mathbf{K}=\left(\begin{array}{ll}
0 & 1 \\
1 & 0
\end{array}\right) .
$$

The symmetry relations (4.15) and (4.16) are used in the appendix to derive the symmetry relations of the one-way analogue of $\underline{\hat{\mathbf{A}}}$. The symmetry relations will be used in section 6 for our final formulation of reciprocity theorems for one-way wave fields, but we will first introduce one-way wave fields in section 5 .

5. One-way wave equation for flux normalized wave fields in SO coordinates. The properties of SO coordinates make the diagonal elements of $\underline{\hat{\mathbf{A}}}$ defined by (4.14a) vanish:

$$
\underline{\hat{\mathbf{A}}}=\left(\begin{array}{cc}
0 & \hat{A}_{12} \\
\underline{\hat{A}}_{21} & 0
\end{array}\right) ;
$$

recall (3.15) and (3.16). Various authors have exploited this antidiagonal structure, for Cartesian coordinates $[4,11]$ and orthogonal coordinates [33], to factorize $\underline{\hat{\mathbf{A}}}$ as

$$
\underline{\hat{\mathbf{A}}}=\underline{\hat{\mathbf{L}}} \underline{\hat{\mathbf{\Lambda}}} \underline{\hat{\mathbf{L}}}^{-1} \text {. }
$$


Here $\underline{\hat{\Lambda}}$ is a diagonal matrix operator, describing one-way wave propagation, while $\underline{\hat{\mathbf{L}}}$ and its inverse $\underline{\hat{\mathbf{L}}}^{-1}$ are, respectively, composition and decomposition operators. Later in this section we give explicit expressions for the diagonalization of $\underline{\hat{\mathbf{A}}}$ formulated by (5.2), based on the antidiagonal structure of $\underline{\hat{\mathbf{A}}}$. Given these expressions we will show that the components of the vector $\underline{\boldsymbol{P}}$,

$$
\underline{\boldsymbol{P}}=\left(\underline{P}^{+}\right)=\underline{\hat{\mathbf{L}}}^{-1} \underline{\boldsymbol{Z}}
$$

can be interpreted as one-way wave fields propagating either in the positive or negative $\underline{\xi}_{3}$ direction; in other words, we will show that (5.3) states directional decomposition in the direction $\underline{\boldsymbol{n}}$. Similarly, the components of the vector

$$
\underline{S}=\left(\underline{S}^{+}\right)=\underline{\hat{\mathbf{L}}}^{-1} \underline{\boldsymbol{D}}
$$

are source fields emitted in either the positive or negative $\underline{\xi}_{3}$-direction.

In terms of these one-way wave fields the wave (4.7) can be rewritten as

$$
\left[\partial_{\xi_{3}}-\underline{\hat{\mathbf{B}}}\right] \underline{\boldsymbol{P}}=\underline{\boldsymbol{S}},
$$

where the one-way operator $\underline{\hat{\mathbf{B}}}$ is given by

$$
\underline{\hat{\mathbf{B}}}=-j \omega \underline{\hat{\boldsymbol{\Lambda}}}+\underline{\hat{\boldsymbol{\Theta}}},
$$

with $\underline{\hat{\boldsymbol{\Theta}}}=-\underline{\hat{\mathbf{L}}}^{-1}\left(\partial_{\underline{\xi}_{3}} \underline{\hat{\mathbf{L}}}\right)$. In the appendix we analyze the symmetry properties of $\underline{\hat{\mathbf{L}}}$ and $\underline{\hat{\mathbf{B}}}$, which is relevant for section 6 .

We formulate our definition of flux normalized wave field decomposition in terms of fractional powers of the operator $\underline{\hat{A}}$ :

$$
\underline{\hat{A}}=\underline{\hat{A}}_{12}^{1 / 2} \underline{\hat{A}}_{21} \underline{\hat{A}}_{12}^{1 / 2} .
$$

The operator $\underline{\hat{A}}$ is symmetric and self-adjoint because its composites are also; recall the definitions (4.14c) and (4.14b). Note that for Cartesian coordinates $\underline{\hat{A}}$ is the Helmholtz operator, for which reason we refer to $\underline{\hat{A}}$ as being of the Helmholtz type. Because the operator $\underline{\hat{A}}_{21}$ is self-adjoint by construction and $\underline{\hat{A}}_{12}$ is exclusively composed of real-valued and positive functions, the operator $\underline{\hat{A}}$ can be represented in terms of its eigenvalues and eigenfunctions $[14,15,16,17]$, from which it is possible to construct the root operators $\underline{\hat{A}}^{1 / 2}$ and $\underline{\hat{A}}^{ \pm 1 / 4}[29]$. These root operators are symmetric but not self-adjoint.

Similar to the diagonalization of matrices with just plain numbers, the diagonalization of matrix operators like $\underline{\hat{\mathbf{A}}}$ defined by (5.1) is not uniquely defined. The remaining degree of freedom is "eigenvector" normalization; see De Hoop [11] or Wapenaar [30] for some choices and their physical interpretations. Here we use flux normalization. A derivation of this normalization from basic principles is beyond the scope of this paper; we just state that (5.2) is satisfied by substitution of

$$
\underline{\hat{\boldsymbol{\Lambda}}}=\underline{\hat{A}}^{1 / 2} \mathbf{J}, \quad \text { where } \quad \mathbf{J}=\left(\begin{array}{cc}
1 & 0 \\
0 & -1
\end{array}\right),
$$

Copyright (c) by SIAM. Unauthorized reproduction of this article is prohibited. 
plus operators for composition,

$$
\underline{\hat{\mathbf{L}}}=2^{-1 / 2}\left(\begin{array}{cc}
\underline{\hat{L}}^{-1} & \underline{\hat{L}}^{-1} \\
\underline{\hat{L}}^{t} & -\underline{\hat{L}}^{t}
\end{array}\right),
$$

and for decomposition,

$$
\underline{\hat{\mathbf{L}}}^{-1}=2^{-1 / 2}\left(\begin{array}{cc}
\underline{\hat{L}} & \underline{\hat{L}}^{t,-1} \\
\underline{\hat{L}} & -\underline{\hat{L}}^{t,-1}
\end{array}\right),
$$

where the basic element $\underline{\hat{L}}$ is given by

$$
\underline{\hat{L}}=\underline{\hat{A}}^{1 / 4} \underline{\hat{A}}_{12}^{-1 / 2} \text {. }
$$

To establish that the combined effect of (5.3), (5.9), and (5.10) indeed represents decomposition into one-way wave fields, we analyze the energy flux in the normal direction $\mathbf{n}$. In the time domain the energy flow is given by the acoustic Poynting vector in that direction, a product of pressure and particle velocity in that direction. As time-domain products do not translate to products in the frequency domain, we analyze the following steady states in the manner of Haines and De Hoop [10]:

$$
\Re\left[\underline{P} e^{j \omega t}\right] \text { and } \Re\left[\underline{V}^{\mathbf{n}} e^{j \omega t}\right] .
$$

Averaged over a time period $2 \pi / \omega$, the steady state Poynting vector reads as

$$
\begin{aligned}
\langle\underline{\boldsymbol{F}} \cdot \underline{\boldsymbol{n}}\rangle_{\text {avg }} & =\int_{0}^{2 \pi / \omega} \Re\left[\underline{P} e^{j \omega t}\right] \Re\left[\underline{V}^{\mathbf{n}} e^{j \omega t}\right] d t \\
& =\left(\underline{P}^{*} \underline{V}^{\mathbf{n}}+\underline{P} \underline{V}^{\mathbf{n}, *}\right) / 4=\underline{\boldsymbol{Z}}^{\dagger} \mathbf{K} \underline{\boldsymbol{Z}} / 4 .
\end{aligned}
$$

For a wave field propagating in the positive $\underline{\xi}_{3}$-direction, $\langle\underline{\boldsymbol{F}} \cdot \underline{\boldsymbol{n}}\rangle_{\text {avg }}$ must be positive. That same wave field should also lead to zero amplitudes for wave fields propagating in the negative $\underline{\xi}_{3}$-direction, or rather $\underline{P}^{-}=0$. In (5.9) the latter condition translates into

$$
\begin{array}{r} 
\\
\underline{\hat{L} P}-\underline{\hat{L}}^{t,-1} \underline{V}^{\mathbf{n}}=0 \\
\Leftrightarrow \underline{V}^{\mathbf{n}}=\underline{\hat{A}}_{12}^{-1 / 2} \underline{\hat{A}}^{1 / 2} \underline{\hat{A}}_{12}^{-1 / 2} P .
\end{array}
$$

To arrive at (5.12), we eliminate $\underline{\hat{L}}$ with (5.10). Upon substitution of (5.12) into (5.11), we see that the two conditions for a wave field propagating in the positive $\underline{\xi}_{3}$-direction, $\langle\underline{\boldsymbol{F}} \cdot \underline{\boldsymbol{n}}\rangle_{\text {avg }} \geq 0$ and $\underline{P}^{-}=0$, can be combined into the inequality

$$
\int_{\underline{\xi}_{3}} u^{\dagger}\left(\underline{\hat{A}}^{1 / 2}+\underline{\hat{A}}^{1 / 2, \dagger}\right) u d^{2} \underline{\boldsymbol{\xi}}_{L} \geq 0
$$

for $u=\underline{\hat{A}}_{12}^{-1 / 2} P$. 


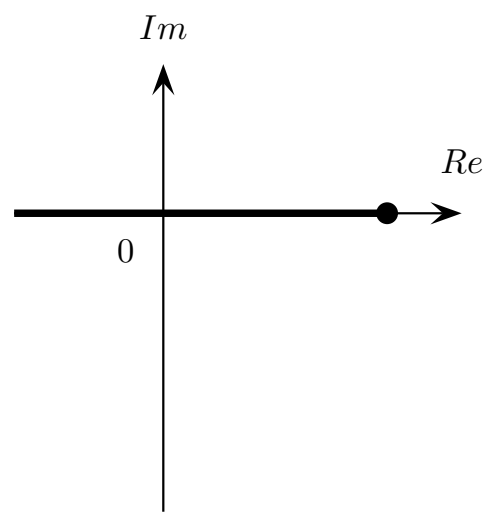

Figure 8. In the complex plane the spectrum of the operator $\underline{\hat{A}}$ is represented by the thick black line.

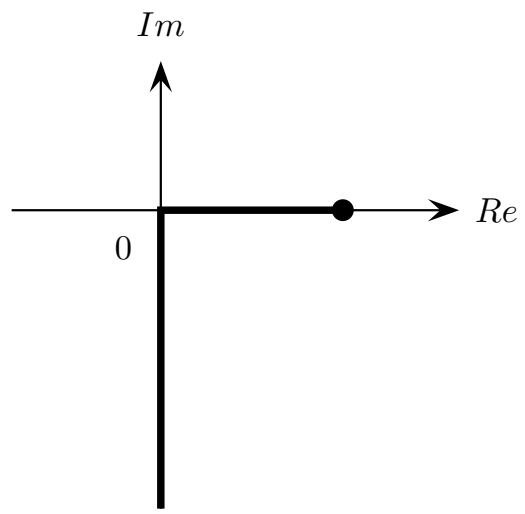

Figure 9. In the complex plane the spectrum of the operator $\underline{\hat{A}}^{1 / 2}$ is represented by the thick black line.

To verify inequality (5.13), we analyze the spectra of $\underline{\hat{A}}$ and $\underline{\hat{A}}^{1 / 2}$. Because $\underline{\hat{A}}$ is self-adjoint, it has real eigenvalues; see Figure 8 for its representation in the complex plane. On the one hand, the negative eigenvalues of $\underline{\hat{A}}$ correspond to imaginary eigenvalues of $\underline{\hat{A}}^{1 / 2}$ and $\underline{\hat{A}}^{1 / 2, \dagger}$; see also Figure 9. These imaginary eigenvalues will always have opposite values, so they do not contribute to the inner product on the left-hand side of inequality (5.13). A positive and real eigenvalue of $\underline{\hat{A}}$, on the other hand, corresponds to positive and real eigenvalues of the square root operator $\underline{\hat{A}}^{1 / 2}$ and its adjoint $\underline{\hat{A}}^{1 / 2, \dagger}$. Hence inequality (5.13) is satisfied so that $\underline{P}^{+}$indeed represents a wave field propagating in the positive $\underline{\xi}_{3}$-direction.

A similar analysis starting from the two conditions $\langle\underline{\boldsymbol{F}} \cdot \underline{\boldsymbol{n}}\rangle_{\text {avg }}<0$ and $\underline{P}^{+}=0$ shows that $\underline{P}^{-}$represents a wave field propagating in the negative $\underline{\xi}_{3}$-direction.

6. Reciprocity theorems. This section describes two different approaches to formulating reciprocity theorems for one-way wave fields. In both approaches we consider two surfaces 
characterized by constant values $\xi_{3}=a, b$ with $a<b$, which we denote by

$$
\mathbb{S}_{c}=\left\{\boldsymbol{\xi} \mid \xi_{3}=c\right\} \quad \text { for } \quad c=a, b .
$$

The volume enclosed by $\mathbb{S}_{a}$ and $\mathbb{S}_{b}$ is denoted by $\mathbb{V}[a, b]$. For volumes and boundary surfaces in LC coordinate systems, Haines and De Hoop [10] already formulated reciprocity theorems in terms of total wave fields. The basis of their convolution-type reciprocity theorems in terms of pressure $P$ and particle velocity $V^{\mathbf{n}}$ is the interaction quantity

$$
\tilde{\boldsymbol{Z}}_{A}^{t} \mathbf{N} \tilde{\boldsymbol{Z}}_{B}=\tilde{P}_{A} \tilde{V}_{B}^{\mathbf{n}}-\tilde{P}_{B} \tilde{V}_{A}^{\mathbf{n}},
$$

relating two states $A$ and $B$. In general the two states have different medium parameters and source functions. In the case of the multiple removal example described in the introduction, state $A$ describes the recorded data with surface-related multiple reflections, and state $B$ the desired multiple-free data; the medium of state $A$ contains the multiple-generating surface, while state $B$ does not. The source functions are taken to be identical. The qualification "convolution type" is inspired by the fact that frequency-domain products in (6.1) correspond to time-domain convolutions. In the same spirit, Haines and De Hoop also formulated correlation-type reciprocity theorems based on the interaction quantity

$$
\tilde{\boldsymbol{Z}}_{A}^{\dagger} \mathbf{K} \tilde{\boldsymbol{Z}}_{B}=\tilde{P}_{A}^{*} \tilde{V}_{B}^{\mathbf{n}}+\tilde{P}_{B} \tilde{V}_{A}^{\mathbf{n}, *}
$$

The reason for analyzing the particular quantities $\tilde{\boldsymbol{Z}}_{A}^{t} \mathbf{N} \tilde{\boldsymbol{Z}}_{B}$ and $\tilde{\boldsymbol{Z}}_{A}^{\dagger} \mathbf{K} \tilde{\boldsymbol{Z}}_{B}$ is that they allow the evaluation of Dirichlet and Neumann conditions. We start from similar interaction quantities for incoming and outgoing boundary conditions on flux normalized wave fields of the convolution type

$$
\underline{\boldsymbol{P}}_{A}^{t} \mathbf{N} \underline{\boldsymbol{P}}_{B}=\underline{P}_{A}^{+} \underline{P}_{B}^{-}-\underline{P}_{A}^{-} \underline{P}_{B}^{+}
$$

and of the correlation type

$$
\underline{\boldsymbol{P}}_{A}^{\dagger} \mathbf{J} \underline{\boldsymbol{P}}_{B}=\underline{P}_{A}^{+, *} \underline{P}_{B}^{+}-\underline{P}_{A}^{-, *} \underline{P}_{B}^{-}
$$

We will use the interaction quantity $\underline{\boldsymbol{P}}_{A}^{t} \mathbf{N} \underline{\boldsymbol{P}}_{B}$ as the basis for reciprocity theorems in terms of flux normalized, one-way wave fields. Integration over the surface $\mathbb{S}_{c}$ makes (6.1) and (6.3) equal except for a minus sign:

$$
\int_{\mathbb{S}_{c}} \underline{\boldsymbol{Z}}_{A}^{t} \mathbf{N} \underline{\boldsymbol{Z}}_{B} d^{2} \boldsymbol{\xi}_{L}=-\int_{\mathbb{S}_{c}} \underline{\boldsymbol{P}}_{A}^{t} \mathbf{N} \underline{\boldsymbol{P}}_{B} d^{2} \boldsymbol{\xi}_{L}
$$

After transforming (6.1) from LC to SO coordinates, the validity of (6.5) can be verified in two steps. First, eliminate both occurrences of $\mathbf{Z}$ with (5.3), through which the operators $\underline{\boldsymbol{L}}^{t}$ and $\underline{\boldsymbol{L}}$ appear, respectively, to the left and right of $\mathbf{N}$. Second, the operators simplify on account of (A.1b). A similar relation can be established between (6.2) and (6.4). One similarly begins by transforming (6.2) from LC to SO coordinates and eliminating both occurrences of $\underline{\boldsymbol{Z}}$ with

Copyright (C) by SIAM. Unauthorized reproduction of this article is prohibited. 
Table 2

State table for $S O$ coordinates in the entire integration volume.

\begin{tabular}{|l|c|c|}
\hline & State A & State B \\
\hline Field & $\underline{\boldsymbol{P}}_{A}$ & $\underline{\boldsymbol{P}}_{B}$ \\
Wave operator & $\underline{\hat{\mathrm{B}}}_{A}$ & $\underline{\hat{\mathrm{B}}}_{B}$ \\
Source & $\underline{\boldsymbol{S}}_{A}$ & $\underline{\boldsymbol{S}}_{B}$ \\
\hline
\end{tabular}

(5.3). The final step in simplifying the operators $\underline{\boldsymbol{L}}^{\dagger}$ and $\underline{\boldsymbol{L}}$ is accomplished by substitution of (A.3b):

$$
\int_{\mathbb{S}_{c}} \mathbf{Z}_{A}^{\dagger} \mathbf{K} \mathbf{Z}_{B} d^{2} \boldsymbol{\xi}_{L} \approx \int_{\mathbb{S}_{c}} \mathbf{P}_{A}^{\dagger} \mathbf{J} \mathbf{P}_{B} d^{2} \boldsymbol{\xi}_{L}
$$

The approximation sign indicates that evanescent wave fields are neglected. This is a common approximation in one-way imaging schemes.

We will first extend the reciprocity theorems for one-way wave fields of Wapenaar [27], which are expressed in terms of Cartesian coordinates and with flat volume boundaries characterized by constant $x_{3}$, to SO coordinates and volume boundaries characterized by constant $\underline{\xi}_{3}$. This requires the existence of a one-to-one map between SO and Cartesian coordinates in the entire integration volume.

For the second approach we will switch between LC and SO coordinates. Analogously to the first approach, we let the volume boundaries coincide with constant values of $\tilde{\xi}_{3}$, but now we apply the wave field decomposition discussed in section 5 to the volume boundaries only. This is possible because at the boundary surfaces $\mathbb{S}_{a}$ and $\mathbb{S}_{b}$ there is the map of LC to SO coordinates implied by (3.17). But inside the integration volume the more flexible LC coordinates will be used. A side effect of the coordinate mapping implied by (3.17) is that at the volume boundaries $\mathbb{S}_{a}$ and $\mathbb{S}_{b}$ the SO-based wave field decomposition of section 5 can be applied directly to wave fields modeled with the LC-based algorithm discussed by Haines and De Hoop [10].

For the states given by Table 2, convolution-type reciprocity theorems can be derived from the interaction quantity $\underline{\boldsymbol{P}}_{A}^{t} \mathbf{N} \underline{\boldsymbol{P}}_{B}$. For a single value $\underline{\xi}_{3}=c$ we integrate the interaction quantity with respect to the lateral variables and differentiate with respect to $\underline{\xi}_{3}$. Substitution of (5.4) for both states $A$ and $B$ then leads to

$$
\begin{aligned}
& \partial_{\underline{\xi}_{3}} \int_{\underline{\mathbb{S}}_{c}} \underline{\boldsymbol{P}}_{A}^{t} \mathbf{N} \underline{\boldsymbol{P}}_{B} d^{2} \underline{\boldsymbol{\xi}}_{L} \\
= & \int_{\underline{\mathbb{S}}_{c}} \underline{\boldsymbol{P}}_{A}^{t}\left[\underline{\hat{\mathbf{B}}}_{A}^{t} \mathbf{N}+\mathbf{N} \underline{\hat{\mathbf{B}}}_{B}\right] \underline{\boldsymbol{P}}_{B} d^{2} \underline{\boldsymbol{\xi}}_{L} \\
& +\int_{\underline{\mathbb{S}}_{c}}\left[\underline{\boldsymbol{S}}_{A}^{t} \mathbf{N} \underline{\boldsymbol{P}}_{B}+\underline{\boldsymbol{P}}_{A}^{t} \mathbf{N} \underline{\boldsymbol{S}}_{B}\right] d^{2} \underline{\boldsymbol{\xi}}_{L} .
\end{aligned}
$$

Note that in the case of identical media the terms proportional to $\underline{\hat{\mathbf{B}}}_{A}^{t}$ and $\underline{\mathbf{B}}_{B}$ cancel on account of (A.4). 
Table 3

State table for $S O$ coordinates on the boundary surfaces.

\begin{tabular}{|l|c|c|}
\hline & State A & State B \\
\hline Field & $\tilde{\boldsymbol{Z}}_{A}$ & $\tilde{\boldsymbol{Z}}_{B}$ \\
Wave operator & $\hat{\tilde{\mathbf{A}}}$ & $\hat{\tilde{\mathbf{A}}}$ \\
Source & $\tilde{\boldsymbol{D}}_{A}$ & $\tilde{\boldsymbol{D}}_{B}$ \\
\hline
\end{tabular}

Further integration of (6.7) over the interval $a \leq \underline{\xi}_{3} \leq b$ leads to a convolution-type reciprocity theorem for one-way wave fields:

$$
\begin{aligned}
\int_{\underline{\mathbb{S}}_{b}} & \underline{\boldsymbol{P}}_{A}^{t} \mathbf{N} \underline{\boldsymbol{P}}_{B} d^{2} \underline{\boldsymbol{\xi}}_{L}-\int_{\underline{\mathbb{S}}_{a}} \underline{\boldsymbol{P}}_{A}^{t} \mathbf{N} \underline{\boldsymbol{P}}_{B} d^{2} \underline{\boldsymbol{\xi}}_{L} \\
= & \int_{\underline{\mathbb{V}[a, b]}} \underline{\boldsymbol{P}}_{A}^{t} \mathbf{N}\left[\underline{\hat{\mathbf{B}}}_{B}-\underline{\hat{\mathbf{B}}}_{A}\right] \underline{\boldsymbol{P}}_{B} d^{3} \underline{\boldsymbol{\xi}} \\
& +\int_{\underline{\mathbb{V}[a, b]}}\left[\underline{\boldsymbol{S}}_{A}^{t} \mathbf{N} \underline{\boldsymbol{P}}_{B}+\underline{\boldsymbol{P}}_{A}^{t} \mathbf{N} \underline{\boldsymbol{S}}_{B}\right] d^{3} \underline{\boldsymbol{\xi}} .
\end{aligned}
$$

Along the same lines the alternative interaction quantity $\underline{\boldsymbol{P}}_{A}^{\dagger} \mathbf{J} \underline{\boldsymbol{P}}_{B}$ leads to a correlation-type reciprocity theorem (correlation-type because the products in this quantity correspond to time-domain correlations). For the constant value $\underline{\xi}_{3}=c$ we have that

$$
\begin{aligned}
& \partial_{\underline{\xi}_{3}} \int_{\underline{\mathbb{S}}_{c}} \underline{\boldsymbol{P}}_{A}^{\dagger} \mathbf{J} \underline{\boldsymbol{P}}_{B} d^{2} \underline{\boldsymbol{\xi}}_{L} \\
= & \int_{\underline{\mathbb{S}}_{c}} \underline{\boldsymbol{P}}_{A}^{\dagger}\left[\underline{\hat{\mathbf{B}}}_{A}^{\dagger} \mathbf{J}+\mathbf{J} \underline{\hat{\boldsymbol{B}}}_{B}\right] \underline{\boldsymbol{P}}_{B} d^{2} \underline{\boldsymbol{\xi}}_{L} \\
& +\int_{\underline{\mathbb{S}}_{c}}\left[\underline{\boldsymbol{S}}_{A}^{\dagger} \mathbf{J} \underline{\boldsymbol{P}}_{B}+\underline{\boldsymbol{P}}_{A}^{\dagger} \mathbf{J} \underline{\boldsymbol{S}}_{B}\right] d^{2} \underline{\boldsymbol{\xi}}_{L} .
\end{aligned}
$$

Similarly as before, integration over the interval $a \leq \underline{\xi}_{3} \leq b$ leads to a correlation-type reciprocity theorem for one-way wave fields:

$$
\begin{aligned}
\int_{\underline{\mathbb{S}}_{b}} \underline{\boldsymbol{P}}_{A}^{\dagger} \mathbf{J} \underline{\boldsymbol{P}}_{B} d^{2} \underline{\boldsymbol{\xi}}_{L}-\int_{\underline{\mathbb{S}}_{a}} \underline{\boldsymbol{P}}_{A}^{\dagger} \mathbf{J} \underline{\boldsymbol{P}}_{B} d^{2} \underline{\boldsymbol{\xi}}_{L} \\
\approx \int_{\underline{\mathbb{V}[a, b]}} \underline{\boldsymbol{P}}_{A}^{\dagger} \mathbf{J}\left[\underline{\hat{\mathbf{B}}}_{B}-\underline{\hat{\mathbf{B}}}_{A}\right] \underline{\boldsymbol{P}}_{B} d^{3} \underline{\boldsymbol{\xi}} \\
\quad+\int_{\underline{\mathbb{V}[a, b]}}\left[\underline{\boldsymbol{S}}_{A}^{\dagger} \mathbf{J} \underline{\boldsymbol{P}}_{B}+\underline{\boldsymbol{P}}_{A}^{\dagger} \mathbf{J} \underline{\boldsymbol{S}}_{B}\right] d^{3} \underline{\boldsymbol{\xi}} .
\end{aligned}
$$

Even for identical medium parameters the operator $\underline{\hat{\mathbf{B}}}_{A}^{\dagger} \mathbf{J}+\mathbf{J} \underline{\hat{\mathbf{B}}}_{B}$ is not exactly zero. Evanescent wave fields have to be neglected for them to cancel; recall (A.9).

Our second approach deals with the states from Table 3 in terms of LC coordinates, with identical medium parameters in both states throughout the integration volume. For volume 
boundaries characterized by either constant $\tilde{\xi}_{3}=a$ or $\tilde{\xi}_{3}=b$, we transform LC into SO coordinates with (3.17). The convenience of this transformation lies in the fact that we can take $\underline{\xi}_{\nu}$ and $\tilde{\xi}_{\nu}$ to be identical in the corresponding hypersurface, so that the lateral differential operators are the same, i.e.,

$$
\partial_{\tilde{\xi}_{\nu}}=\partial_{\underline{\xi}_{\nu}}
$$

The lateral dependencies of scalar functions such as the medium parameters $\tilde{\rho}, \tilde{K}$ and, more importantly, the modeling results $\tilde{P}$ and $\tilde{V}^{\mathbf{n}}$, are therefore not affected by transitions from $\mathrm{LC}$ coordinates to the $\mathrm{SO}$ coordinates as specified above: on the volume boundaries

$$
\underline{P}=\tilde{P} \quad \text { and } \quad \underline{V}^{\mathbf{n}}=\tilde{V}^{\mathbf{n}} .
$$

In the manner of Haines and De Hoop [10] we consider the two states from Table 3 which correspond to the same medium. This time we integrate the interaction quantity $\tilde{\boldsymbol{Z}}_{A}^{t} \mathbf{N} \tilde{\boldsymbol{Z}}_{B}$ over $\tilde{\boldsymbol{\xi}}_{L}$ and differentiate with respect to $\tilde{\xi}_{3}$. Now we use (4.7) to eliminate $\partial_{\xi_{3}} \tilde{\boldsymbol{Z}}_{A}$ and $\partial_{\xi_{3}} \tilde{\boldsymbol{Z}}_{B}$ and write

$$
\begin{aligned}
& \partial_{\tilde{\xi}_{3}} \int_{\underline{\mathbb{S}}_{c}} \tilde{\boldsymbol{Z}}_{A}^{t} \mathbf{N} \tilde{\boldsymbol{Z}}_{B} d^{2} \tilde{\boldsymbol{\xi}}_{L} \\
= & -j \omega \int_{\underline{\mathbb{S}}_{c}} \tilde{\boldsymbol{Z}}_{A}^{t}\left[\hat{\tilde{\mathbf{A}}}^{t} \mathbf{N}+\mathbf{N} \hat{\tilde{\mathbf{A}}}\right] \tilde{\boldsymbol{Z}}_{B} d^{2} \tilde{\boldsymbol{\xi}}_{L} \\
& +\int_{\underline{\mathbb{S}}_{c}}\left[\tilde{\boldsymbol{D}}_{A}^{t} \mathbf{N} \tilde{\boldsymbol{Z}}_{B}+\tilde{\boldsymbol{Z}}_{A}^{t} \mathbf{N} \tilde{\boldsymbol{D}}_{B}\right] d^{2} \tilde{\boldsymbol{\xi}}_{L}
\end{aligned}
$$

The terms proportional to $\hat{\tilde{\mathbf{A}}}^{t}$ and $\hat{\tilde{\mathbf{A}}}$ cancel on account of (4.15). Next we integrate (6.11) with respect to $\tilde{\xi}_{3}$ on the interval $[a, b]$; its left-hand side leads to two boundary terms, one for each limit of the interval. Similarly to (6.5), the boundary terms can be expressed as

$$
\int_{\underline{\mathbb{S}}_{c}} \tilde{\boldsymbol{Z}}_{A}^{t} \mathbf{N} \tilde{\boldsymbol{Z}}_{B} d^{2} \tilde{\boldsymbol{\xi}}_{L}=-\int_{\underline{\mathbb{S}}_{c}} \tilde{\boldsymbol{P}}_{A}^{t} \mathbf{N} \tilde{\boldsymbol{P}}_{B} d^{2} \tilde{\boldsymbol{\xi}}_{L}
$$

for $c=a, b$. Note that on account of our choice of SO coordinates given by (3.17), there is no need to distinguish between $\tilde{\xi}_{\nu}$ and $\underline{\xi}_{\nu}$. Hence, for LC coordinates we have the convolution-type reciprocity theorem

$$
\begin{aligned}
& \int_{\underline{\mathbb{S}}_{a}} \tilde{\boldsymbol{P}}_{A}^{t} \mathbf{N} \tilde{\boldsymbol{P}}_{B} d^{2} \tilde{\boldsymbol{\xi}}_{L}-\int_{\underline{\mathbb{S}}_{b}} \tilde{\boldsymbol{P}}_{A}^{t} \mathbf{N} \tilde{\boldsymbol{P}}_{B} d^{2} \tilde{\boldsymbol{\xi}}_{L} \\
= & \int_{\underline{\mathbb{V}}[a, b]}\left[\tilde{\boldsymbol{D}}_{A}^{t} \mathbf{N} \tilde{\boldsymbol{Z}}_{B}+\tilde{\boldsymbol{Z}}_{A}^{t} \mathbf{N} \tilde{\boldsymbol{D}}_{B}\right] d^{3} \tilde{\boldsymbol{\xi}} .
\end{aligned}
$$

If the source fields $\tilde{\boldsymbol{D}}_{A, B}$ are zero inside the integration volume, then the volume integral containing the source terms vanishes. So after evaluating the matrix-vector products in (6.13) with $\tilde{\boldsymbol{P}}^{t}=\left(\tilde{P}^{+}, \tilde{P}^{-}\right)$and the definition $\mathbf{N}$ from (4.15), the ingoing and outgoing wave fields 
at the boundaries $\tilde{\xi}_{3}=a$ and $\tilde{\xi}_{3}=b$ are related by

$$
\begin{aligned}
& \int_{\underline{\mathbb{S}}_{b}}\left[\tilde{P}_{A}^{+} \tilde{P}_{B}^{-}-\tilde{P}_{A}^{-} \tilde{P}_{B}^{+}\right] d^{2} \tilde{\boldsymbol{\xi}}_{L} \\
= & \int_{\underline{\mathbb{S}}_{a}}\left[\tilde{P}_{A}^{+} \tilde{P}_{B}^{-}-\tilde{P}_{A}^{-} \tilde{P}_{B}^{+}\right] d^{2} \tilde{\boldsymbol{\xi}}_{L} .
\end{aligned}
$$

Correlation-type reciprocity theorems are based on the interaction quantity $\tilde{\boldsymbol{Z}}_{A}^{\dagger} \mathbf{K} \tilde{\boldsymbol{Z}}_{B}$. The terms proportional to $\hat{\tilde{\mathbf{A}}}^{\dagger}$ and $\hat{\tilde{\mathbf{A}}}$ cancel on account of (4.16). After integration over $\tilde{\xi}_{3}$ on the interval $[a, b]$, the boundary terms can be approximated by

$$
\int_{\tilde{\xi}_{3}=c} \tilde{\boldsymbol{Z}}_{A}^{\dagger} \mathbf{K} \tilde{\boldsymbol{Z}}_{B} d^{2} \tilde{\boldsymbol{\xi}}_{L} \approx \int_{\tilde{\xi}_{3}=c} \tilde{\boldsymbol{P}}_{A}^{\dagger} \mathbf{J} \tilde{\boldsymbol{P}}_{B} d^{2} \tilde{\boldsymbol{\xi}}_{L},
$$

similar to (6.6). The approximation in (6.15) is exclusively related to neglecting evanescent wave fields. In the case of identical media, correlation-type reciprocity theorems for source-free integration volumes therefore read as

$$
\begin{aligned}
& \int_{\underline{\mathbb{S}}_{b}}\left[\left(\tilde{P}_{A}^{+}\right)^{*} \tilde{P}_{B}^{+}-\left(\tilde{P}_{A}^{-}\right)^{*} \tilde{P}_{B}^{-}\right] d^{2} \tilde{\boldsymbol{\xi}}_{L} \\
\approx & \int_{\underline{\mathbb{S}}_{a}}\left[\left(\tilde{P}_{A}^{+}\right)^{*} \tilde{P}_{B}^{+}-\left(\tilde{P}_{A}^{-}\right)^{*} \tilde{P}_{B}^{-}\right] d^{2} \tilde{\boldsymbol{\xi}}_{L} .
\end{aligned}
$$

As $\underline{\hat{A}}$ defined by (5.6), (4.14b), and (4.14c) is a pseudodifferential operator, our approach of flux normalized decomposition is valid only if $f$ in (3.10) varies smoothly as a function of $x_{1}$ and $x_{2}$; see, for example, Van den Berg and De Hoop [25]. For our first approach this requirement is necessary on the entire integration volume, but for our second approach it is necessary only on the volume boundaries. An additional requirement is that there must be a reversible mapping between Cartesian and LC/SO coordinates. Recall Figure 5 for an example that does not have such a reversible mapping.

7. Discussion and conclusions. The one-way reciprocity theorems, formulated by (6.8) and (6.10), are general relations between one-way wave fields, sources, and medium parameters in two different states. Originally they were formulated in Cartesian coordinates, and wave field decomposition (also known as wave splitting) was restricted to flat planes. After specifying curvilinear coordinates in section 3, this paper describes in sections 4 and 5 how the restriction can be relaxed. The reciprocity theorems formulated in section 6 allow for the formulation of imaging and inverse scattering algorithms in terms of one-way wave fields on domains delimited by nonflat boundaries. Geophysical applications whose validity is extended to SO coordinates through (6.8) and (6.10) include inversion based on generalized primary representations (Wapenaar [28]), prediction and elimination of multiple reflections (Verschuur, Berkhout, and Wapenaar [26], Van Borselen, Fokkema, and Van den Berg [24], Amundsen [1], Wapenaar, Thorbecke, and Draganov [32], and Frijlink, Van Borselen, and Sollner [6]), and synthesis of unrecorded data (Wapenaar, Thorbecke, and Draganov [32], Schuster et al. [19], Snieder, Sheiman, and Calvert [20], and Wapenaar, Slob, and Snieder [31]). 
As discussed in section 3.3, SO coordinates are characterized by their basis vectors; they do not offer an actual coordinate system. This can be remedied by tying SO to LC coordinates. An inherent, additional limitation is the requirement of smoothly varying medium parameters, density, and compression modulus, throughout the integration volume, due to the involvement of pseudodifferential operators. LC coordinates allow this requirement to be relaxed if

- the medium parameters can be taken to be identical in both states and

- decomposition into incoming and outgoing wave fields is required only at the boundaries of the integration volume. The fact remains that at these boundaries smoothly varying medium parameters and conformity to interfaces in the subsurface are required. They can be positioned only in between these interfaces, avoiding intersection with the major discontinuities. However, in the interior of the integration volume these smoothness conditions are no longer required.

Appendix. Symmetry properties of the one-way wave equation for flux normalized wave fields. As stated before, the directional decomposition defined by (5.7)-(5.10) is not unique. Other choices for the "eigenvector" normalization [11] could have satisfied the argument of (5.11)-(5.13) equally well. Our motivation for using flux normalized decomposition stems from its symmetry properties; see De Hoop [11]. The definitions (5.8) and (5.9) of the composition and decomposition operators imply that they are interrelated by

$$
\begin{aligned}
\mathbf{N} \underline{\mathbf{L}}^{t} \mathbf{N} & =\underline{\hat{\mathbf{L}}}^{-1} \\
\Leftrightarrow & \underline{\hat{\mathbf{L}}}{ }^{t} \underline{\mathbf{N}}=-\mathbf{N} .
\end{aligned}
$$

To arrive at (A.1b) we multiplied (A.1a) from the left with $\mathbf{N}^{-1}=-\mathbf{N}$ and from the right with $\underline{\hat{\mathbf{L}}}$. In section 6 we use these relations for convolution-type reciprocity theorems. Their correlation-type counterparts are based on adjoint instead of transposed operators. However, to obtain correlation-type analogues of the relations (A.1) it is necessary to neglect evanescent wave fields. This approximation means that

$$
\left\{\underline{\hat{A}}^{ \pm 1 / 4}\right\}^{\dagger}=\left\{\underline{\hat{A}}^{ \pm 1 / 4}\right\}^{*} \approx \underline{\hat{A}}^{ \pm 1 / 4}
$$

(see Wapenaar and Grimbergen [29]). All further approximation signs occurring in this paper arise from neglecting evanescent wave fields, an approximation that is routinely followed in most seismic, one-way imaging schemes. With this approximation the (de)composition operators are also interrelated by

$$
\begin{aligned}
\mathbf{J} \underline{\hat{\mathbf{L}}}^{\dagger} \mathbf{K} & \approx \underline{\hat{\mathbf{L}}}^{-1} \\
\Leftrightarrow & \underline{\hat{\mathbf{L}}}{ }^{\dagger} \mathbf{K} \underline{\hat{\mathbf{L}}} \approx \mathbf{J} .
\end{aligned}
$$

The one-way operator $\underline{\mathbf{B}}$ defined by (5.5) has the same symplectic property as $\underline{\hat{\mathbf{A}}}$, that is,

$$
\underline{\hat{\mathbf{B}}}^{t} \mathbf{N}=-\mathbf{N} \underline{\hat{\mathbf{B}}} \text {. }
$$

We prove (A.4) separately for the two terms $j \omega \underline{\hat{\Lambda}}$ and $\underline{\hat{\Theta}}$. For the former, matrix operator (A.4) is satisfied because $\underline{\hat{\Lambda}}$ is symmetric and diagonal. For the latter, we first note that the 
product rule for differentiation applied to $\partial_{\underline{\xi}_{3}}\left(\underline{\hat{\mathbf{L}}}^{-1} \underline{\hat{\mathbf{L}}}\right)=0$ yields

$$
\begin{aligned}
\underline{\hat{\mathbf{\Theta}}}=-\underline{\hat{\mathbf{L}}}^{-1}\left(\partial_{\underline{\xi}_{3}} \underline{\hat{\mathbf{L}}}\right)=\left(\partial_{\underline{\xi}_{3}} \underline{\hat{\mathbf{L}}}^{-1}\right) \underline{\hat{\mathbf{L}}} \\
\Leftrightarrow \quad \underline{\hat{\mathbf{\Theta}}}^{t}=\underline{\hat{\mathbf{L}}}^{t}\left(\partial_{\underline{\xi}_{3}} \underline{\hat{\mathbf{L}}}^{-1, t}\right) .
\end{aligned}
$$

To arrive at (A.5) we used the property that differentiation of $\underline{\hat{\mathbf{L}}}$ with respect to $\underline{\xi}_{3}$ commutes with transposition, since the operator character of $\underline{\hat{\mathbf{L}}}$ is exclusively related to $\underline{\xi}_{1,2}$. Next we use (A.1a) to eliminate $\underline{\hat{\mathbf{L}}}^{ \pm 1, t}$ on the right-hand side of (A.5) in favor of $\underline{\hat{\mathbf{L}}}^{\mp 1}$. After multiplication of the result from the right with $\mathbf{N}$, the observation that $\mathbf{N}^{2}$ equals the identity matrix allows us to conclude that

$$
\underline{\hat{\Theta}}^{t} \mathbf{N}=-\mathbf{N} \underline{\hat{\Theta}}
$$

by which (A.4) has been proved.

For correlation-type reciprocity theorems an adjoint-based analogue of (A.4) is necessary. Its derivation is similar to that of (A.4), but it involves (A.3) instead of (A.1), and as a result, evanescent wave fields are neglected. First we substitute (5.2) into (4.16). After left and right multiplication with $\underline{\hat{\mathbf{L}}}^{\dagger}$ and $\underline{\hat{\mathbf{L}}}$, respectively, substitution of (A.3b) yields

$$
\begin{aligned}
\underline{\hat{\boldsymbol{\Lambda}}}^{\dagger} \mathbf{J} & \approx \mathbf{J} \underline{\hat{\Lambda}} \\
\Leftrightarrow(j \omega \underline{\hat{\Lambda}} & \dagger \\
\mathbf{J} & \approx-j \omega \mathbf{J} \underline{\hat{\Lambda}} .
\end{aligned}
$$

For $\underline{\hat{\boldsymbol{\Theta}}}$ we multiply the complex conjugate of $\underline{\hat{\boldsymbol{\Theta}}}^{t}$ from the right with $\mathbf{J}$. On account of (A.5) we can use (A.3a) to eliminate the adjoints $\underline{\hat{\mathbf{L}}}^{ \pm 1, \dagger}$ in favor of $\underline{\hat{\mathbf{L}}}^{\mp 1}$. Since $\mathbf{J}^{2}$ and $\mathbf{K}^{2}$ are both equal to identity, the elimination result reads as

$$
\underline{\hat{\Theta}}^{\dagger} \mathbf{J} \approx-\mathbf{J} \underline{\hat{\Theta}} .
$$

Equations (A.7) and (A.8) can finally be combined into

$$
\underline{\hat{\mathbf{B}}}^{\dagger} \mathbf{J} \approx-\mathbf{J} \underline{\hat{\mathbf{B}}} .
$$

The symmetry properties (A.1b), (A.3b), (A.4), and (A.9) are used in section 6 .

Acknowledgments. The authors want to thank Dr. John Haines plus three anonymous reviewers. Their comments and suggestions helped to focus the paper.

\section{REFERENCES}

[1] L. Amundsen, Elimination of free-surface related multiples without need of the source wavelet, Geophysics, 66 (2001), pp. 327-341.

[2] Y. M. Berezansky, Z. G. Sheftel, and G. F. Us, Functional Analysis, Vol. I, Oper. Theory Adv. Appl. 85, Birkhäuser Verlag, Basel, 1996. 
[3] Y. M. Berezansky, Z. G. Sheftel, And G. F. Us, Functional Analysis, Vol. II, Oper. Theory Adv. Appl. 86, Birkhäuser Verlag, Basel, 1996.

[4] J. W. Evans, Splitting methods and invariant imbedding for time-independent wave propagation in focusing media and wave guides, J. Math. Phys., 29 (1988), pp. 97-102.

[5] J. T. Fokkema And P. M. VAn Den Berg, Seismic Applications of Acoustic Reciprocity, Elsevier, Amsterdam, 1993.

[6] M. O. Frijlink, R. G. van Borselen, and W. Sollner, On the free surface assumption for marine data driven demultiple methods, in Extended Abstracts, 71st EAGE Conference and Exhibition, Amsterdam, 2009.

[7] M. Frijlink And K. WapenaAR, Migration with correction for transmission losses in arbitrary homogeneous media, in SEG Expanded Abstracts, Vol. 32, Society of Exploration Geophysicists, 2004, pp. 1029-1032.

[8] Y. C. Fung And P. Tong, Classical and Computational Solid Mechanics, World Scientific, Singapore, 2001.

[9] M. Gustafsson, Wave Splitting in Direct and Inverse Scattering Problems, Ph.D. thesis, Lund University, Lund, Sweden, 2000.

[10] A. J. Haines AND M. V. DE Hoop, An invariant imbedding analysis of general wave scattering problems, J. Math. Phys., 37 (1996), pp. 3854-3881.

[11] M. V. DE Hoop, Generalization of the Bremmer coupling series, J. Math. Phys., 37 (1996), pp. 3246-3282.

[12] B. L. N. Kennett, Seismic Wave Propagation in Stratified Media, Cambridge University Press, Cambridge, UK, 1983.

[13] W. A. Mulder, Rigorous redatuming, Geophys. J. Int., 161 (2005), pp. 401-415.

[14] M. Reed And B. Simon, Methods of Modern Mathematical Physics. I. Functional Analysis, Academic Press, New York, 1972.

[15] M. Reed And B. Simon, Methods of Modern Mathematical Physics. II. Fourier Analysis, SelfAdjointness, Academic Press, New York, 1975.

[16] M. Reed And B. Simon, Methods of Modern Mathematical Physics. III. Scattering Theory, Academic Press, New York, 1979.

[17] M. Reed And B. Simon, Methods of Modern Mathematical Physics. IV. Analysis of Operators, Academic Press, New York, 1978.

[18] P. Sava And S. Fomel, Riemannian wave field extrapolation, Geophysics, 70 (2005), pp. T45-T56.

[19] G. T. Schuster, J. Yu, J. Sheng, and J. Rickett, Interferometric/daylight seismic imaging, Geophys. J. Int., 157 (2004), pp. 838-852.

[20] R. Snieder, J. Sheiman, And R. Calvert, Equivalence of the virtual-source method and wave-field deconvolution in seismic interferometry, Phys. Rev. E, 73 (2006), 066620.

[21] J. W. Strutt (Baron Rayleigh), The Theory of Sound, Vol. II, 2nd ed., Macmillan, New York, 1896.

[22] Z. Sun, Reconstruction of source and medium parameters via wave-splitting and Green function equations, SIAM J. Appl. Math., 56 (1996), pp. 1146-1163.

[23] B. URsin, Review of elastic and electromagnetic wave propagation in horizontally layered media, Geophysics, 48 (1983), pp. 1063-1081.

[24] R. G. van Borselen, J. T. Fokkema, And P. M. van Den Berg, Surface-related multiple elimination, Geophysics, 61 (1996), pp. 202-210.

[25] P. M. van den Berg AND A. T. DE Hoop, Reflection and transmission of electromagnetic waves at a rough interface between two different media, IEEE Trans. Geosci. Remote Sensing, GE-22 (1984), pp. $42-52$.

[26] D. J. Verschuur, A. J. Berkhout, and C. P. A. Wapenaar, Adaptive surface-related multiple elimination, Geophysics, 57 (1992), pp. 1166-1177.

[27] C. P. A. WapenaAR, Reciprocity theorems for two-way and one-way wave vectors: A comparison, J. Acoust. Soc. Am., 100 (1996), pp. 3508-3518.

[28] C. P. A. WapenaAR, One-way representations of seismic data, Geophys. J. Int., 127 (1996), pp. 178-188.

[29] C. P. A. Wapenaar and J. L. T. Grimbergen, Reciprocity theorems for one-way wave fields, Geophys. J. Int., 127 (1996), pp. 169-177.

[30] K. WAPENAAR, Reciprocity properties of one-way propagators, Geophysics, 63 (1998), pp. 1795-1798.

[31] K. WapenaAR, E. Slob, And R. Snieder, Seismic and electromagnetic controlled-source interferometry in dissipative media, Geophys. Prosp., 56 (2008), pp. 419-434.

Copyright (C) by SIAM. Unauthorized reproduction of this article is prohibited. 
[32] K. Wapenaar, J. Thorbecke, and D. Draganov, Relations between reflection and transmission responses of three-dimensional inhomogeneous media, Geophys. J. Int., 156 (2004), pp. 179-194.

[33] V. H. Weston, Factorization of the wave equation in a nonplanar stratified medium, J. Math. Phys., 29 (1988), pp. 36-45.

[34] V. H. Weston AND S. He, Wave splitting of the telegraph equation in $\mathbf{R}^{3}$ and its application to inverse scattering, Inverse Problems, 9 (1993), pp. 789-812.

Copyright @ by SIAM. Unauthorized reproduction of this article is prohibited. 